\title{
Article \\ The Effect of Cognitive Behavioural Therapy for Insomnia (CBT-I) on Subjective-Objective Sleep Discrepancy in Individuals with Co-Morbid Insomnia and Sleep Apnoea: A Randomised Controlled Trial
}

\author{
Darah-Bree Bensen-Boakes ${ }^{1, *}$, Amal Osman ${ }^{2}$, Leon Lack ${ }^{1}$, Peter Catcheside ${ }^{2}$, Nick Antic ${ }^{2,3,+}$, Simon S. Smith ${ }^{4}$ (D, \\ Ching Li Chai-Coetzer ${ }^{2,3}$, Amanda O'Grady ${ }^{2}$, Nicola Dunn ${ }^{5}$, Jan Robinson ${ }^{5}$, Doug McEvoy ${ }^{2}$ and \\ Alexander Sweetman ${ }^{2}$ (D)
}

\section{check for}

updates

Citation: Bensen-Boakes, D.-B.;

Osman, A.; Lack, L.; Catcheside, P.; Antic, N.; Smith, S.S.; Chai-Coetzer, C.L.; O'Grady, A.; Dunn, N.; Robinson, J.; et al. The Effect of Cognitive Behavioural Therapy for Insomnia (CBT-I) on

Subjective-Objective Sleep Discrepancy in Individuals with Co-Morbid Insomnia and Sleep Apnoea: A Randomised Controlled Trial. Appl. Sci. 2022, 12, 1787. https://doi.org/10.3390/app12041787

Academic Editor: Raúl Quevedo-Blasco

Received: 17 December 2021 Accepted: 4 February 2022

Published: 9 February 2022

Publisher's Note: MDPI stays neutral with regard to jurisdictional claims in published maps and institutional affiliations.

Copyright: (C) 2022 by the authors. Licensee MDPI, Basel, Switzerland. This article is an open access article distributed under the terms and conditions of the Creative Commons Attribution (CC BY) license (https:// creativecommons.org/licenses/by/ $4.0 /)$.
1 College of Education, Psychology and Social Work, Flinders University, Bedford Park, SA 5042, Australia; leon.lack@flinders.edu.au

2 Adelaide Institute for Sleep Health, Flinders Health and Medical Research Institute: Sleep Health, College of Medicine and Public Health, Flinders University, Bedford Park, SA 5042, Australia; amal.osman@flinders.edu.au (A.O.); peter.catcheside@flinders.edu.au (P.C.); chingli.chai-coetzer@sa.gov.au (C.L.C.-C.); mandy.ogrady@flinders.edu.au (A.O.); doug.mcevoy@flinders.edu.au (D.M.); alexander.sweetman@flinders.edu.au (A.S.)

3 Sleep Health Service, Respiratory and Sleep Services, Southern Adelaide Local Health Network, Adelaide, SA 5000, Australia

4 Institute for Social Science Research (ISSR), The University of Queensland, Brisbane, QLD 4068, Australia; simon.smith@uq.edu.au

5 Thoracic Program, The Prince Charles Hospital, Brisbane, QLD 4032, Australia; nicola.dunn@health.qld.gov.au (N.D.); jan.robinson2@health.qld.gov.au (J.R.)

* Correspondence: darahbree.bensenboakes@flinders.edu.au

+ Author Deceased.

\begin{abstract}
People with insomnia frequently underestimate the duration of their sleep compared to objective polysomnography-measured sleep duration. Cognitive behavioural therapy for insomnia (CBT-I) is the most effective treatment for insomnia and also reduces the degree of sleep underestimation. Obstructive sleep apnoea (OSA) is a highly prevalent sleep disorder characterised by frequent narrowing (hypopnoea) and closure (apnoea) of the upper airway during sleep. Comorbid insomnia and sleep apnoea (COMISA) is a prevalent and debilitating disorder. No study has investigated subjectively (sleep diary) versus objectively (polysomnography) measured sleep discrepancies (SOSD) in individuals with COMISA before or following CBT-I. This randomised waitlist-controlled trial investigated SOSD in 145 participants with COMISA before and 6-weeks after CBT-I $(n=72)$ versus control $(n=73)$. All participants were studied prior to continuous positive airway pressure treatment for sleep apnoea. At baseline, participants underestimated their total sleep time (mean $\pm \mathrm{SD}-51.9 \pm 94.1 \mathrm{~min}$ ) and sleep efficiency $(-9.6 \pm 18.3 \%)$, and overestimated sleep onset latency ( $34.5 \pm 86.1 \mathrm{~min}$; all $p=<0.001$ ). Mixed models indicated a main effect of time on reduction of SOSD in both groups, but no between-group difference in the reduction of any SOSD parameters. These findings may indicate that untreated OSA contributes to a discrepancy between perceived and objective sleep parameters in people with COMISA that is not amenable to CBT-I alone (ACTRN12613001178730).
\end{abstract}

Keywords: insomnia; obstructive sleep apnoea; CBT-I; subjective-objective sleep discrepancy

\section{Introduction}

Chronic insomnia and obstructive sleep apnoea (OSA) are the two most common sleep disorders and frequently co-occur [1,2]. Chronic insomnia is characterised by self-reported difficulty initiating sleep, maintaining sleep, and/or early morning awakenings, combined with daytime impairments that persist for at least 3 months $[1,3,4]$. Chronic insomnia occurs 
in approximately $6-15 \%$ of the general population $[4,5]$. Obstructive sleep apnoea (OSA) is characterised by frequent narrowing (hypopnoea) and closure (apnoea) of the upper airway during sleep [6]. These events cause hypoxemia, cortical arousals and awakenings from sleep, and daytime impairments [6]. Moderate OSA occurs in approximately 6-17\% of the general population [7].

Co-morbid insomnia and sleep apnoea (COMISA) is a prevalent and debilitating sleep disorder that is more difficult to treat compared to either insomnia or OSA alone $[2,8]$. For example, approximately $30-40 \%$ of people with insomnia have co-morbid OSA and $30-50 \%$ of sleep clinic patients with OSA report insomnia symptoms [9,10]. COMISA is associated with worse sleep, increased daytime impairments, poorer mental and physical health, and reduced longevity, compared to people with neither disorder or those with insomnia or OSA alone [8,11,12].

People with insomnia alone generally underestimate their sleep time, overestimate how long it takes to initially fall asleep, and overestimate the time they spend awake throughout the night, compared to polysomnography-derived estimates of these parameters [13]. This phenomenon is referred to as subjective-objective sleep discrepancy (SOSD, but also "sleep misperception", "sleep underestimation", and "sleep-state discrepancy"), and may contribute to the development and maintenance of insomnia [14-17]. For example, Edinger and Fins (1995) [18] and Crönlein et al. (2019) [14] found that individuals with insomnia underestimated total sleep time by approximately 50 and $100 \mathrm{~min}$, respectively [18].

Cognitive behavioural therapy for insomnia (CBT-I) is an effective and recommended "first-line" treatment for insomnia [19]. CBT-I is a multi-component therapy including behavioural, cognitive, and education components typically delivered over $4-8$ weekly treatment sessions [19-22]. Furthermore, CBT-I in patients with insomnia alone leads to large improvements in subjective sleep estimates and improvements in assessments of daytime functioning and feelings, despite little change in objective total sleep time. This results in a convergence of the two measures post-treatment, and a reduction in SOSD, meaning that subjective and objective measures align more closely [14,23-25]. However, few studies have investigated SOSD in patients with COMISA and no study has investigated the effect of CBT-I on SOSD in a COMISA sample.

SOSD may reflect bi-directional associations between insomnia and OSA [9]. For example, periods of brief sleep and awakenings following respiratory events may be (mis)perceived as periods of prolonged wakefulness [13]. Perceptions of long periods of wakefulness may increase frustration and cognitive arousal, contributing to an increased duration of light sleep [9]. In turn, increased time in light and transitional sleep may increase the likelihood of upper airway collapse [26,27], and exacerbation of each condition [9]. Bianchi et al. (2013) [28] investigated total sleep time SOSD in 92 people with insomnia alone, 66 with OSA alone, and 119 with COMISA and found the insomnia alone group significantly under-estimated total sleep time compared to objective recordings (67 $\pm 87 \mathrm{~min})$, but the OSA alone (17 $\pm 78 \mathrm{~min})$ and COMISA groups $(36 \pm 88 \mathrm{~min}) \mathrm{did}$ not [28]. Similarly, Choi et al. (2016) [29] found that an insomnia alone group showed greater SOSD than an OSA alone group but not a COMISA group [29]. In a comparison of COMISA, insomnia alone, OSA alone, and no insomnia/OSA groups, Ma et al. (2021) [30] recently found COMISA participants to have the highest average levels of total sleep time underestimation and sleep onset latency overestimation. These markers of sleep misperception were also significantly greater compared to the OSA alone group, and significant relationships between SOSD and reduced quality of life were also observed in those with insomnia and COMISA [30]. Overall, Ma et al. (2021) [30] concluded that insomnia (whether insomnia alone or COMISA) plays a significant role in SOSD of sleep parameters.

No study to date has investigated the effect of CBT-I on SOSD in people with COMISA to help clarify the extent to which insomnia and/or OSA may contribute to perceptual sleep time discrepancies. Such a study could help to resolve the relative contributions of insomnia and OSA mechanisms to this COMISA cohort. This study aimed to use RCT data to investigate patterns of SOSD at baseline in people with COMISA, exploratory 
associations between SOSD and other sleep, daytime function, and sociodemographic information at baseline, and changes in SOSD following a CBT-I program, versus notreatment control.

\section{Materials and Methods}

\subsection{Participants}

The study included 145 participants with comorbid insomnia and OSA (COMISA) from a recent RCT [31] (Figure 1). Participants were recruited at two locations: the Adelaide Institute for Sleep Health in South Australia and The Prince Charles Hospital in Queensland, Australia. Community advertisement was also used as a recruitment tool. All participants were diagnosed with insomnia (psychologist diagnosis of insomnia according to ICSD-3 criteria [1]), and OSA (apnoea-hypopnoea index (AHI) $\geq 15$ events/h sleep, according to an overnight polysomnography study). Individuals were excluded from the study if they had another sleep or medical disorder that required treatment, history of continuous positive airway pressure (CPAP) therapy use in the last four months, current participation in another research study, significant language barrier, or if they lived in a remote location precluding study visit attendance [31]. The study had Human Research Ethics Committee approval and all participants provided informed written consent.

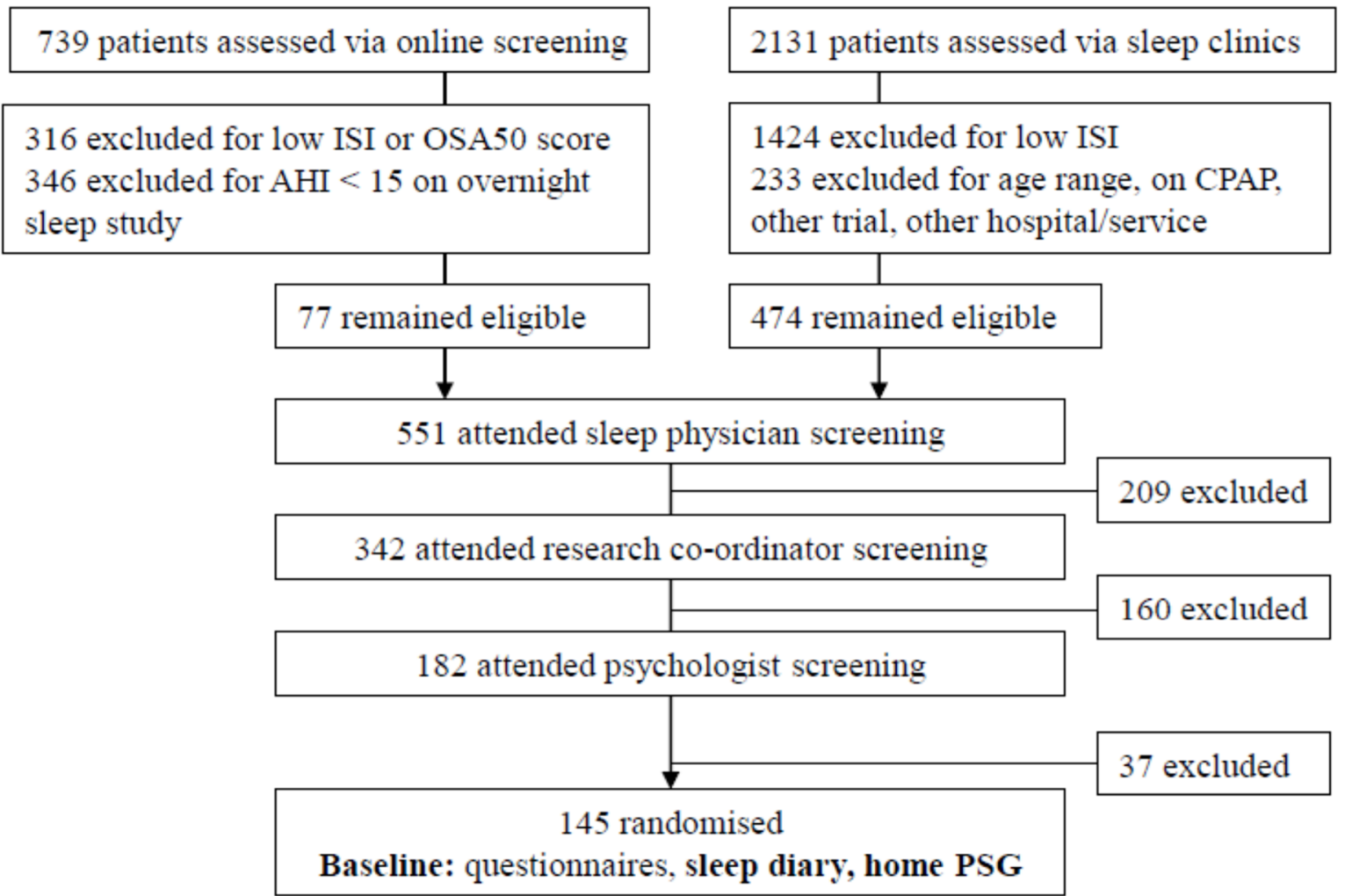

Figure 1. Study screening, flow, and retention. Adapted from Sweetman et al. [31].

\subsection{Procedure}

Participants completed baseline questionnaires, a one-week sleep diary, and overnight polysomnography including a next-morning sleep diary, before being randomly allocated to a 4-session CBT-I program, or a no-treatment control group. Participants completed the same battery of questionnaires, sleep diary, overnight polysomnography, and overnight sleep diary at 6-weeks follow-up (post CBT-I/control). Although participants were involved in a larger RCT to investigate the effect of CBT-I versus control on subsequent adherence to CPAP (CPAP was administered after the 6-week follow-up time point), postCPAP data were not included in the present study. CBT-I content, delivery, efficacy, and 
treatment fidelity have been described previously [31]. Briefly, a manualised program was delivered by psychologists over the course of 4-weekly individual or small-group sessions. Participants were provided feedback on their own baseline polysomnography compared to overnight diary parameters during the second session to highlight potential episodes of SOSD.

\subsection{Clinical Trials Registration}

This study was registered via the clinical trials register. "Treating comorbid insomnia with obstructive sleep apnoea (COMSIA) study: A new treatment strategy for patients with combined insomnia and sleep apnoea, https://www.anzctr.org.au/Trial/ Registration/TrialReview.aspx?id=365184 Australian New Zealand Clinical Trials Registry: ACTRN12613001178730. Universal Trial Number: U1111-1149-4230." (accessed on 1 March 2021).

\subsection{Protocol}

\subsubsection{Home-Based Sleep Studies and SOSD Calculation}

All participants completed home-based polysomnography studies at baseline (before CBT-I/waitlist control randomisation) and 6-week follow-up. Participants were set up with recording equipment at sleep laboratories (the Adelaide Institute for Sleep Health and the Queensland University of Technology) by trained sleep technologists. Two electroencephalogram (C3 to M2 and C4 to M1), one electromyogram, two electrooculogram, and two leg electromyogram channels were recorded. Finger pulse oximeters, nasal cannulas, oro-nasal thermistor, and chest and abdominal bands recorded oxygen saturation, flow, and respiratory effort. Trained technicians scored the home studies according to American Academy of Sleep Medicine (2007) [32] "alternate" criteria for respiratory events for AHI scores, using an AHI cut-off $\geq 15 / \mathrm{h}$ to define OSA [32,33]. The trained technicians were blind to participants' condition. The sleep window on the polysomnography was based on a combination of participant self-reported lights off/on time, and objectively derived measures (e.g., position sensor reading, attachment time of oximeter). On the same night as the polysomnography study, each participant self-reported time in bed, time out of bed, total sleep time, sleep onset latency, and wake after sleep onset on an overnight sleep-wake diary. Sleep efficiency was calculated as the percentage of time in bed spent asleep.

Overnight diary parameters were compared to polysomnography parameters to calculate SOSD for total sleep time, sleep onset latency, wake after sleep onset, and sleep efficiency at baseline and 6-week follow-up. SOSD was calculated by subtracting the polysomnography measures from the overnight sleep diary measures (subjective minus objective) for each sleep/wake parameter. For example, if a participant reported a total sleep time of $420 \mathrm{~min}$, but had an objective total sleep time of $480 \mathrm{~min}$, their total sleep time SOSD would be $-60 \mathrm{~min}$. A sensitivity definition of SOSD was used to align with previous literature, in which SOSD was dichotomously defined as overestimation or underestimation of each parameter (total sleep time, wake after sleep onset, and sleep onset latency) by at least $60 \mathrm{~min}$ [34].

\subsubsection{Questionnaire Battery}

A questionnaire battery including measures of sleep and daytime functioning was completed at baseline and 6-week follow-up. The "insomnia severity index", is a 7-item self-report questionnaire measure of global insomnia severity extensive used in insomnia and COMISA research [35]. Scores range from 0-28, with higher scores indicating greater insomnia severity [35]. The 12-item "daytime feelings and functioning scale" was used to assess impaired mood, social, and cognitive function experienced in the last two weeks [36]. Scores range from 0-36, with higher scores indicating greater daytime impairment [36]. The 7-item "Flinders fatigue scale" was used to measure fatigue experienced over the last two weeks [37]. Total scores range from 0-31, with higher scores reflecting more daytime fatigue [38]. Depression, anxiety, and stress across the past week were assessed using 
the 21-item "depression, anxiety and stress sale" (DASS) [39], where sub-scores range from $0-42$, with higher scores representing greater severity of depression, anxiety, and stress symptoms, respectively [40]. Daytime sleepiness was assessed using the 8-item "Epworth sleepiness scale" (ESS) [41] where scores range from 0 (no sleepiness) to 24 (highest sleepiness) [42]. Finally, the 16-item "dysfunctional beliefs and attitudes about sleep scale" (DBAS) was used to assess thoughts related to sleep/insomnia [43]. There are four subsets of the DBAS: (1) misattribution/amplification of consequences, (2) irrational sleep worries, (3) unrealistic expectations about sleep, and (4) dysfunctional beliefs about medications, with scores ranging from 0 (lowest level of dysfunctional beliefs) to 100 (highest level) [43].

\subsection{Statistical Analyses}

All data were analysed with SPSS (IBM, version 25). Visual interpretation of SOSD histograms indicated normally distributed variables. A small number of extreme outliers (see Figure 2) were removed in sensitivity analyses which did not substantially change results. Paired-samples t-tests were used to compare subjective and objective sleep parameters before treatment. Linear mixed models investigated the effect of group (CBT-I, control) and time (baseline, 6-week follow-up) on change in SOSD parameters (total sleep time, sleep onset latency, wake after sleep onset, and sleep efficiency). Correlations and independent-samples t-tests were used to investigate relationships between SOSD and other sociodemographic, sleep, and daytime functioning variables at baseline. A two-tailed alpha $<0.05$ was considered statistically significant. All data are reported as mean \pm SDs (baseline analyses) and mean $\pm 95 \%$ confidence intervals (95\% CI; longitudinal analyses), unless otherwise indicated. SOSD was recalculated as a ratio using the index from Manconi et al. (2010) [44]; results can be found in the Supplementary Materials.

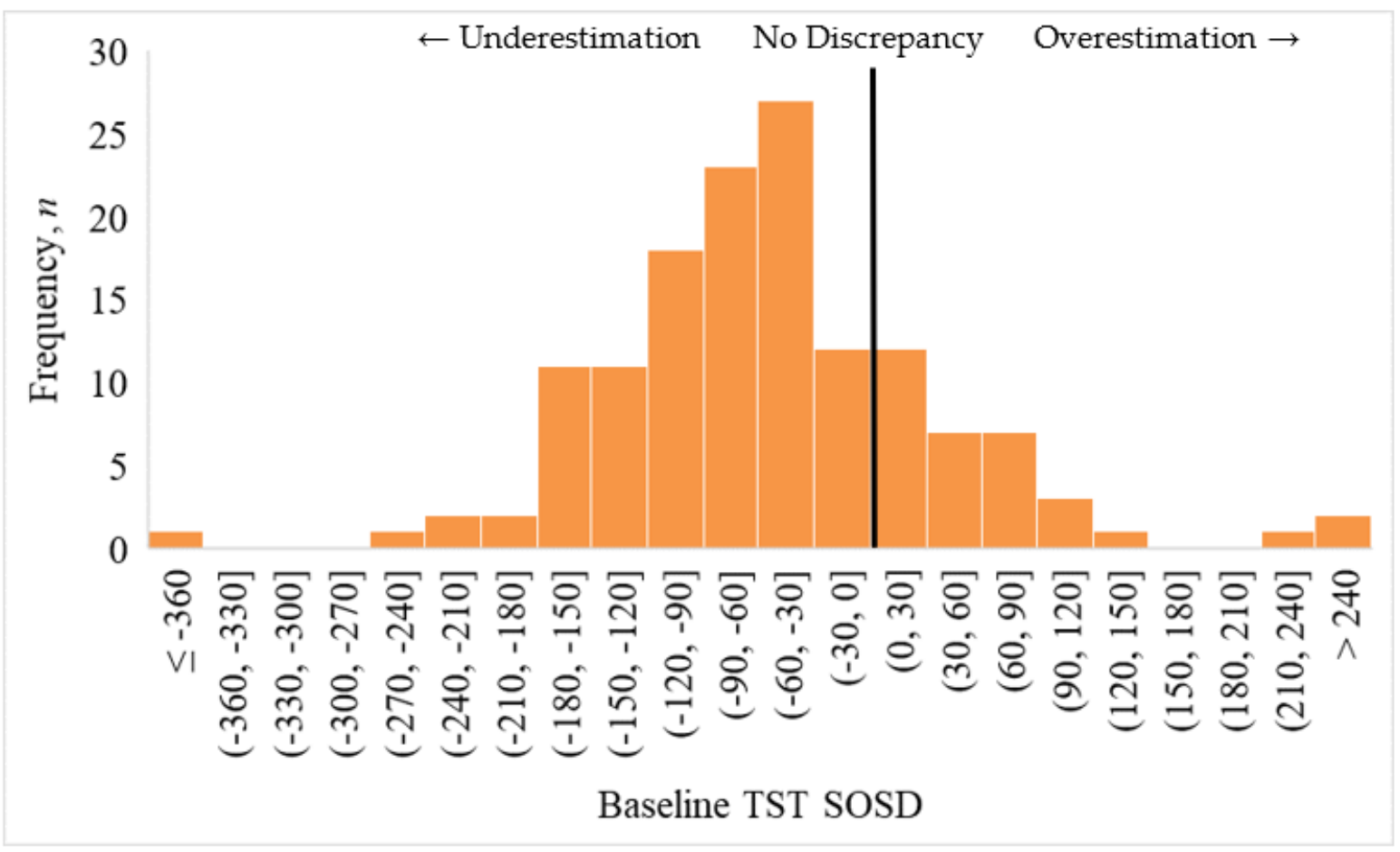

Figure 2. Histogram of total sleep time (TST) SOSD at baseline.

\section{Results}

\subsection{Participant Characteristics at Baseline}

Participant characteristics at baseline and comparisons between the CBT-I and control group are presented in Table 1 . There were no significant between-group differences in any 
socio-demographic, objective, or subjective sleep parameters, or questionnaire measures at baseline.

Table 1. Baseline participant characteristics (M (SD); or $n(\%))$.

\begin{tabular}{ccccc}
\hline & $\begin{array}{c}\text { Total } \\
(\boldsymbol{n}=\mathbf{1 4 5})\end{array}$ & $\begin{array}{c}\text { Control } \\
(\boldsymbol{n}=\mathbf{7 3})\end{array}$ & $\begin{array}{c}\text { CBT-I } \\
(\boldsymbol{n}=\mathbf{7 2})\end{array}$ & $p$ \\
\hline Age & $58.2(9.9)$ & $57.3(9.9)$ & $59.1(9.9)$ & 0.279 \\
Sex, \% & $55.2 \%$ male, & $54.8 \%$ male, & $55.6 \%$ male, & 0.927 \\
BMI $\left(\mathrm{kg} \cdot \mathrm{m}^{-2}\right)$ & $44.8 \%$ female & $45.2 \%$ female & $44.4 \%$ female & $*$ \\
AHI (events $/ \mathrm{h})$ & $35.1(6.4)$ & $35.9(6.6)$ & $34.4(6.1)$ & 0.171 \\
Insomnia severity index & $34.5(21.9)$ & $35.8(23.9)$ & $33.2(19.8)$ & 0.471 \\
Flinders fatigue scale & $18.2(5.0)$ & $17.9(4.7)$ & $18.5(5.4)$ & 0.497 \\
Epworth sleepiness scale & $15.9(6.3)$ & $15.9(5.8)$ & $16.0(6.8)$ & 0.969 \\
Daytime feelings and & $9.3(4.8)$ & $9.7(4.5)$ & $8.9(5.0)$ & 0.335 \\
functioning scale & $17.4(8.3)$ & $17.8(8.5)$ & $16.9(8.0)$ & 0.551 \\
DBAS & $59.4(17.0)$ & $58.6(15.4)$ & $60.3(18.5)$ & 0.549 \\
Depression & $14.6(12.1)$ & $14.6(12.8)$ & $14.5(11.4)$ & 0.959 \\
Anxiety & $9.6(8.3)$ & $8.9(7.9)$ & $10.2(8.8)$ & 0.352 \\
Stress & $15.4(9.6)$ & $15.6(9.8)$ & $15.1(9.5)$ & 0.792 \\
One-week diary & & & & \\
Total sleep time (min) & $348.4(77.5)$ & $350.6(9.5)$ & $346.2(8.8)$ & 0.730 \\
Sleep onset latency (min) & $53.0(42.9)$ & $49.6(4.1)$ & $56.6(5.9)$ & 0.326 \\
Wake after sleep onset (min) & $96.9(66.5)$ & $99.3(8.5)$ & $94.5(7.1)$ & 0.665 \\
Sleep efficiency $(\%)$ & $67.4(13.4)$ & $68.0(1.7)$ & $66.7(1.5)$ & 0.582
\end{tabular}

AHI, apnoea-hypopnoea index; BMI, body mass index; DBAS, dysfunctional beliefs and attitudes about sleep scale. ${ }^{*}, \mathrm{Chi}^{2}$ test.

\subsection{SOSD at Baseline}

SOSD parameters for the whole group at baseline are presented in Table 2. Compared to polysomnography measures, participants self-reported significantly lower total sleep time and sleep efficiency, and greater sleep onset latency. For average wake after sleep onset, no significant difference between polysomnography measures and self-reported measures were observed. To replicate the findings of Liu et al. 2020, the percentage of participants who underestimated total sleep time and overestimated wake after sleep onset, and sleep onset latency by $60 \mathrm{~min}$ or more was calculated. Underestimations of total sleep time by $\geq 60 \mathrm{~min}$ accounted for $51.8 \%$, underestimation of sleep onset latency by $\geq 60 \mathrm{~min}$ accounted for $20.7 \%$, and $19.3 \%$ overestimated and $20 \%$ underestimated wake after sleep onset by $\geq 60 \mathrm{~min}$. A histogram of total sleep time discrepancy for the whole sample is presented in Figure 2. There were no statistically significant between-group differences in any SOSD parameter at baseline (all $p>0.174)$.

Table 2. Baseline subjective and objective sleep parameters, and discrepancy (SOSD) for total sleep time, sleep onset latency, wake after sleep onset, and sleep efficiency.

\begin{tabular}{cccccc}
\hline & Subjective & Objective & Cohen's $d$ & $p$ & SOSD \\
\hline Total sleep time (min) & $314.9(98.0)$ & $366.7(86.7)$ & 0.56 & $<0.001$ & $-51.9(94.1)$ \\
Sleep onset latency (min) & $65.2(80.7)$ & $30.1(50.3)$ & 0.52 & $<0.001$ & $34.5(86.1)$ \\
Wake after sleep onset (min) & $91.8(69.6)$ & $95.6(57.1)$ & 0.06 & 0.524 & $-4.2(76.9)$ \\
Sleep efficiency (\%) & $64.9(18.1)$ & $74.7(13.0$ & 0.62 & $<0.001$ & $-9.6(18.3)$ \\
\hline
\end{tabular}

\subsection{The Effect of CBT-I, versus Control, on Subjective-Objective Discrepancy}

There was no significant group by time interaction on total sleep time SOSD (Figure 3; $\mathrm{F}(1,135)=0.156, p=0.693)$. There were also no significant group by time interactions on sleep onset latency, wake after sleep onset, or sleep efficiency SOSD (all interaction $p \geq 0.347$ ). There was a significant main effect of time on total sleep time SOSD indicating that discrepancy reduced from baseline $(\mathrm{M}=-51.78,95 \% \mathrm{CI}=-66.29,-37.27)$ to 6-week 
follow-up for both groups $(\mathrm{M}=-21.40,95 \% \mathrm{CI}=-36.15,-6.65 ; \mathrm{F}(1,135)=11.93, p=0.001)$. There were also main effects of time on sleep onset latency SOSD and sleep efficiency SOSD, but not for wake after sleep onset SOSD. There was no significant main effect of group on total sleep time SOSD, indicating that total sleep time SOSD did not differ between the CBT-I group and the control group when collapsed over time $(\mathrm{F}(1,135)=1.98, p=0.162)$. There were also no significant main effects of group on sleep onset latency SOSD, wake after sleep onset SOSD, or sleep efficiency SOSD (see Supplementary Materials).

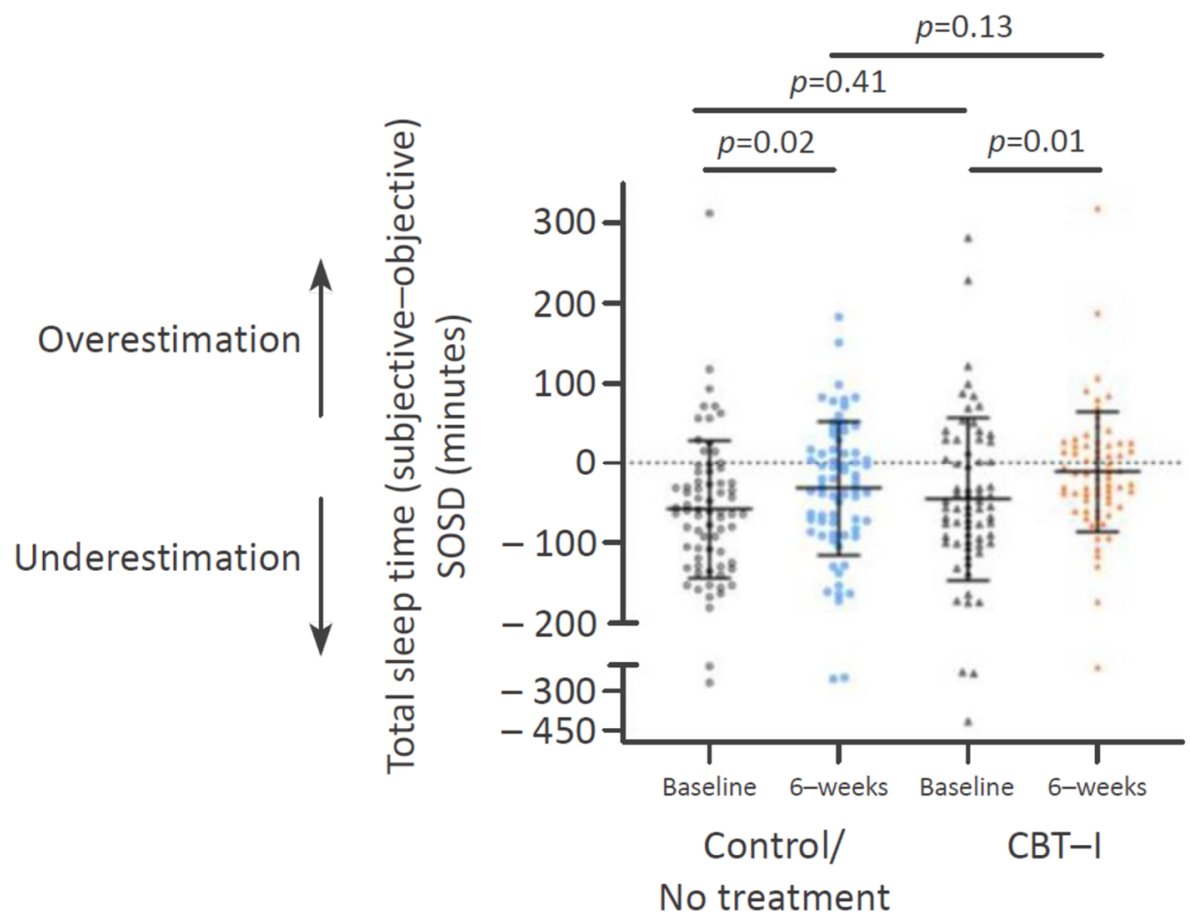

Figure 3. Between-group change in mean ( \pm standard deviation) total sleep time (TST) subjectiveobjective discrepancy from baseline to 6-week follow-up.

\subsection{Relationships between SOSD and Baseline Socio-Demographic, Questionnaire, and Sleep Data}

Given the increased likelihood of type 1 error due to the large number of exploratory analyses, the handful of statistically significant associations presented below should be interpreted with caution, and in the context of weak-to-moderate effect sizes. DBAS (expectations subscale) and wake after sleep onset SOSD were negatively correlated $(r=-0.216$, $p=0.010)$, indicating that wake after sleep onset overestimation was associated with lower DBAS (expectations). However, none of the SOSD measures were related to any socio-demographic, PSG, insomnia severity or other daytime impairment scales (see Supplementary Materials Tables S3-S7).

There was no relationship between overall the apnoea-hypopnoea index (AHI) and any SOSD parameter at baseline. Stage one sleep (N1) AHI and wake after sleep onset SOSD were negatively correlated $(r=-0.195, p=0.021)$, indicating that greater wake after sleep onset overestimation was associated with lower AHI in N1 sleep. N2 AHI and sleep onset latency SOSD were positively correlated $(\mathrm{r}=0.190, p=0.025)$, indicating that greater sleep onset latency underestimation was associated with a lower AHI in N2 sleep. REM AHI and total sleep time SOSD were positively correlated $(r=0.198 . p=0.022)$, indicating that greater overestimation of total sleep time was associated with a higher AHI in REM sleep. Arousal index scores and wake after sleep onset SOSD were negatively correlated $(\mathrm{r}=-0.243, p=0.01)$, indicating that greater wake after sleep onset overestimation was associated with lower arousal index scores. Minutes in N2 sleep was positively correlated with wake after sleep onset SOSD $(r=0.229, p=0.006)$, and negatively correlated with both total sleep time SOSD $(r=-0.362, p<0.001)$ and sleep efficiency SOSD $(r=-0.304$, 
$p<0.001$ ), indicating that greater underestimation of sleep time was associated with more N2 sleep. Minutes in REM sleep was negatively correlated with both total sleep time $\operatorname{SOSD}(r=-0.295, p \leq 0.001)$ and sleep efficiency SOSD $(r=-0.209, p=0.013)$, indicating that greater total sleep time and sleep efficiency underestimation is associated with more minutes in REM sleep. Removal of extreme outliers (Figure 2) did not substantially change the pattern of results described above.

\section{Discussion}

This is the first RCT to examine the effect of CBT-I, versus control, on subjectiveobjective sleep discrepancy in individuals with COMISA. At baseline, individuals with COMISA underestimated total sleep time by an average of $52 \mathrm{~min}$, and overestimated sleep onset latency by 35 min compared to polysomnography measurements, with no consistent relationships between SOSD parameters and any sociodemographic, sleep, or daytime function measures. In this COMISA group it has previously been shown that CBT-I significantly improves $(p<0.001)$ insomnia severity (measured by the "insomnia severity index") (baseline $\mathrm{M}=18.5, \mathrm{SD}=1.3$; 6-weeks after CBT-I $\mathrm{M}=12.2, \mathrm{SD}=1.4$ ) compared to the waitlist control group (baseline $\mathrm{M}=18.0, \mathrm{SD}=1.3$; 6-weeks after control $\mathrm{M}=16.6, \mathrm{SD}=1.4$ ) [31]. However, unlike previous findings in those with insomnia alone, this improvement in sleep was not associated with a specific improvement in SOSD in people with COMISA.

Previous studies investigating baseline SOSD in COMISA groups have reported inconsistent findings. Similar to Choi et al. (2016) [29], we showed participants with COMISA significantly underestimated total sleep time and overestimated sleep onset latency before treatment [29]. However, our findings differ from other studies which have reported no or minimal subjective-objective discrepancy of sleep duration in COMISA $[28,30]$. This may be due to differences in study samples or methodology. For example, we recruited COMISA patients through specialist sleep clinics, while Ma et al. (2021) [30] examined a population-based sample $(\mathrm{N}=638)$. Furthermore, Bianchi et al. (2013) [28] examined subjective-objective discrepancy during laboratory-based polysomnography. Contrastingly, our polysomnography studies were conducted in participants' home environment. This is more directly relevant to insomnia-related sleep perceptions, timing, and duration of objective recording, and discrepancy between parameters when compared to in laboratory studies [45].

Choi et al. (2016) [29] reported overestimation of sleep onset latency in participants with insomnia alone (17.4 min), COMISA (14.5 min), and OSA alone (15 min). In contrast, a larger average sleep onset latency overestimation of $34.5 \mathrm{~min}$ was seen in our COMISA sample. Liu et al. (2020) [34] found that among 355 patients with OSA, 35.5\% overestimated sleep duration by $60 \mathrm{~min}$ or more, and $20 \%$ underestimated sleep duration by $60 \mathrm{~min}$ or more. In our COMISA sample, $9.9 \%$ of participants overestimated sleep duration by $\geq 60 \mathrm{~min}$ and $51.8 \%$ underestimated sleep duration by $\geq 60 \mathrm{~min}$ suggestive of a potential tendency towards underestimating total sleep time in individuals with COMISA compared to individuals with OSA alone in other studies.

Previous studies have reported that CBT-I reduces the discrepancy between subjective and objective measures of sleep in individuals with insomnia alone $[14,23,25,46]$. This is likely driven by a large improvement in subjective sleep estimates, and minimal change in objective sleep during treatment that results in a convergence of the two measures post CBT-I-treatment but could also be explained by regression to the mean [23,24]. Crönlein et al. [14] and Janků et al. [25] did not have control groups, and Kay et al. [46] had a control group whereby the participants did not have insomnia ("good sleepers"), meaning that these studies were not RCTs with a sensible comparator group. Whereas the current study was a RCT and therefore it importantly controlled for temporal effects independent of CBT-I. The results of the current study show that total sleep time, sleep onset latency, and sleep efficiency, but not wake after sleep onset, SOSD declined over time with no differences between CBT-I and control groups. These findings indicate the importance of a waitlist 
control group and could indicate regression to the mean, first night effects, or perhaps a learning effect where studies that draw participant attention to sleep variables may influence subjective responses over time, irrespective of treatment interventions. These results contrast with previous studies in insomnia samples that found evidence for a reduction of SOSD after CBT-I [14,23,25,47,48].

The lack of a CBT-I effect may be due to the shorter four-session CBT-I intervention used in the current study, as each of the above-mentioned studies included CBT-I programs with more than four sessions. Indeed, Chan et al. (2021) [49] found no effect of a brief behavioural therapy for insomnia, versus active control on changes in total sleep time or wake after sleep onset SOSD from baseline to post-treatment (CBT-I)/waitlist control period [49]. Furthermore, Kay et al. [46] investigated the effect of eight sessions of CBT-I for older adults with insomnia $(\mathrm{N}=63)$ and compared this to a sample of older adults with good sleep who did not receive CBT-I $(\mathrm{N}=51)$. Kay et al. [46] found no group by time interaction on discrepancy, suggesting that CBT-I was not effective at reducing the SOSD in an older adult insomnia sample.

It is possible that SOSD results partly from insomnia and partly for OSA-specific factors in people with COMISA [9]. This may explain the absence of an effect of CBT-I, versus control on reducing SOSD parameters. As this is the first study conducted that investigated the effectiveness of CBT-I versus control as a tool for reducing SOSD in COMISA, there have been no other studies looking at the timing of CBT-I and treatment of OSA. For example, future studies could compare groups that receive CBT-I before CPAP, CPAP before CBT-I, or CBT-I and CPAP simultaneously to determine which combination most effectively reduces SOSD. This would help address the question of the disorder-specific maintenance of SOSD in people with COMISA.

Similarly to previous research with people with insomnia, we found few associations between baseline variables and SOSD in COMISA patients [18]. A small number of weak and inconsistent associations were observed between SOSD parameters and other questionnaire and sleep study parameters, including associations between higher stage-specific AHI (in N1, N2, and REM) and greater underestimation of sleep parameters. Given the exploratory nature of this cross-sectional aim and the large number of statistical tests conducted, it is likely that the handful of statistically significant correlations occurred due to type one error. This study supports evidence from studies of participants with insomnia alone [18] that baseline sociodemographic characteristics, sleep, and daytime impairments are not consistently associated with SOSD in individuals with COMISA.

This study should be interpreted in light of some limitations. Firstly, this study only used four sessions of CBT-I, compared to the traditionally recommended 6-8 sessions of CBT-I. Previous studies have found CBT-I of more than four sessions to be effective at reducing SOSD in insomnia alone samples $[14,23,25,47,48]$. Thus, future studies should investigate the effect of 6-8 session CBT-I programs on SOSD in people with COMISA.

Secondly, the focus of this study was on COMISA where potentially useful comparisons with insomnia alone or OSA alone groups were not available (e.g., [28]). Given the reported effectiveness of CBT-I in improving sleep estimates in patients with insomnia alone, future studies should compare the effect of CBT-I on SOSD between individuals with COMISA and those with insomnia alone, to investigate a potential moderation effect of co-morbid OSA on change in SOSD. CBT-I is clearly useful and beneficial to individuals with COMISA [31], but the results of this study suggest that a focus on SOSD reduction as an outcome when using CBT-I in individuals with COMISA may not be fruitful.

Finally, the AASM alternate criteria for scoring hypopnoeas (50\% reduction in airflow with $\geq 3 \%$ oxygen desaturations or arousal) were used to score AHI (and consequently trial eligibility). Using these criteria may increase the likelihood of a bias towards a stronger association between hypopnoeas and awakenings, and perceiving prior sleep as wakefulness. This may limit the generalisability of these findings to studies with previous hypopnoea scoring criteria (i.e., without arousals). 


\section{Conclusions}

This study investigated patterns of subjective-objective sleep discrepancy at baseline and following CBT-I, versus control, in participants with COMISA. Participants significantly underestimated sleep duration and sleep efficiency and overestimated sleep onset latency at baseline compared to polysomnography. However, there was no difference in any SOSD changes over time between the CBT-I and the control group to support CBT-I effects on subjective-objective sleep discrepancy. There were also no consistent associations between SOSD parameters and any baseline subjective or objective sleep, daytime function, or socio-demographic factors. Further investigation into the aetiology of SOSD in COMISA, and the effect of CBT-I and CPAP therapy on SOSD in individuals with insomnia alone, OSA alone, and COMISA are warranted.

Supplementary Materials: There are supplementary materials that accompany this report. The supplementary materials file contains; average SOSD ratio data between groups and time, average SOSD data between groups and time, and correlation analyses between SOSD parameters and sociodemographic variables, sleep diary parameters, questionnaire parameters, and polysomnography parameters at baseline. The following supporting information can be downloaded at: https: / / www.mdpi.com/article/10.3390/app12041787/s1, Table S1: Average SOSD ratio between groups and time ( $\pm 95 \%$ confidence intervals); Table S2a. Complete case analysis, average SOSD between groups and time (standard deviation); Table S2b. ITT Linear Mixed Models results, average SOSD between groups and time ( $\pm 95 \%$ confidence intervals); Table S3. Correlations between stage-specific, AHI, arousal index and polysomnography sleep duration with SOSD parameters at baseline; Table S4: Correlations between SOSD parameters and sociodemographic variables at baseline; Table S5: Correlations between SOSD parameters and 7-day sleep diary variables at baseline; Table S6. Correlations between SOSD parameters and questionnaire battery variables at baseline; Table S7. Correlations between SOSD parameters and polysomnography variables at baseline.

Author Contributions: Conceptualisation, D.-B.B.-B., A.O. (Amal Osman), L.L., and A.S.; formal analysis, D.-B.B.-B., A.O. (Amal Osman), L.L., and A.S.; funding acquisition, L.L., P.C., N.A., S.S.S., C.L.C.-C., and D.M.; methodology, D.-B.B.-B., A.O. (Amal Osman), L.L., P.C., N.A., S.S.S., C.L.C.-C., A.O. (Amanda $\mathrm{O}^{\prime} \mathrm{Grady}$ ), N.D., J.R., D.M., and A.S.; project administration, L.L., P.C., N.A., S.S.S., C.L.C.-C., A.O. (Amanda O'Grady), N.D., J.R., D.M., and A.S.; supervision, A.O. (Amal Osman), L.L., and A.S.; writing-original draft, D.-B.B.-B., A.O. (Amal Osman), L.L., and A.S.; writing-review and editing, D.-B.B.-B., A.O. (Amal Osman), L.L., P.C., S.S.S., C.L.C.-C., A.O. (Amanda O'Grady), N.D., J.R., D.M., and A.S. All authors have read and agreed to the published version of the manuscript.

Funding: This research was funded by a National Health and Medical Research Council Grant (1049591; Treating insomnia co-morbid with obstructive sleep apnoea: A randomised controlled clinical effectiveness trial).

Institutional Review Board Statement: This research was approved by the Southern Adelaide Clinical Human Research Ethics Committee (428.12; South Australian Local Health Network, Flinders University of South Australia), the Human Research Ethics Committee (1300000302; The Prince Charles Hospital, Brisbane), the Queensland University of Technology Human Research Ethics Committee, and the External Request Evaluation Committee (Department of Human Services, Australia). Treating comorbid insomnia with obstructive sleep apnoea (COMSIA) study: A new treatment strategy for patients with combined insomnia and sleep apnoea, https://www.anzctr.org.au/Trial/ Registration/TrialReview.aspx?id=365184 Australian New Zealand Clinical Trials Registry: ACTRN12613001178730. Universal Trial Number: U1111-1149-4230 (accessed on 1 March 2021).

Informed Consent Statement: Informed consent was obtained from all subjects involved in the study.

Data Availability Statement: Data have not been made publicly available as participants did not provide informed consent for data to be shared publicly. 


\begin{abstract}
Acknowledgments: The authors would like to acknowledge the following people for their contributions to this study: Ashliegh Perry, Neralie Cain, Melissa Wilson, Sara Winter, and Lynette Buller for delivering the Cognitive Behavioral Therapy sessions; Laura Bandick, Michaela O'Keefe, Hiro Tojo, and Sharn Rowland for administering CPAP education and setups; Emer Van Ryswyk, Cassandra Pattinson, and Alicia Allan for managing recruitment and home studies; Tim Jarryd, Alistair Edwards, Henry Scown, Hayden Ng, Gorica Micic, Kalina Rossa, Sherrie-Anne Kaye, and Luisa Roeder for assistance with sleep studies; Carl Downey for scoring sleep studies; Air Liquide for supplying CPAP equipment and assisting with CPAP setups; NHMRC for funding this research. James Douglas and Denzil Paul for participant recruitment and study co-ordination.
\end{abstract}

Conflicts of Interest: The authors declare no conflict of interest. Financial disclosure: D.-B.B.-B., A.O. (Amal Osman), A.S., S.S., A.O. (Amanda O'Grady), N.D., J.R., C.L.C.-C., and P.W. have no financial information to disclose. D.M. reports research funding support from Philips Respironics. P.C. received salary support via an Australian Research Council Future Fellowship (FT120100510) and reports research funding support from Philips Respironics via the CRC for Alertness, Safety, and Productivity. L.L. has received funding support from Re-timer (Re-timer Pty Ltd., Adelaide, Australia). Nonfinancial disclosure: D.-B.B.-B., A.O. (Amal Osman), A.S., S.S., A.O. (Amanda O'Grady), N.D., J.R., P.W., and L.L. have no non-financial information to disclosure. D.M. reports research equipment donations from ResMed and Air Liquide. P.C. reports equipment support from Philips Respironics and Air Liquide. CLCC reports previous equipment support from Philips Respironics and ResMed (ApneaLink devices and CPAP equipment).

\title{
References
}

1. Sateia, M.J. International Classification of Sleep Disorders. Chest 2014, 146, 1387-1394. [CrossRef] [PubMed]

2. Sweetman, A.M.; Lack, L.C.; Catcheside, P.; Antic, N.A.; Chai-Coetzer, C.L.; Smith, S.; Douglas, J.A.; McEvoy, D. Developing a successful treatment for co-morbid insomnia and sleep apnoea. Sleep Med. Rev. 2017, 33, 28-38. [CrossRef] [PubMed]

3. Morin, M.C.; Benca, R. Chronic insomnia. Lancet 2012, 379, 1129-1141. [CrossRef]

4. Reynolds, A.C.; Appleton, S.L.; Gill, T.K.; Adams, R.J.; Sa, W. Chronic Insomnia Disorder in Australia; Sleep Health Foundation: Arlington, VA, USA, 2019.

5. Ohayon, M.M. Epidemiology of insomnia: What we know and what we still need to learn. Sleep Med. Rev. 2002, 6, 97-111. [CrossRef] [PubMed]

6. Gottlieb, D.J.; Punjabi, N.M. Diagnosis and management of obstructive sleep apnea: A review. JAMA 2020, 323, 1389-1400. [CrossRef]

7. Senaratna, C.V.; Perret, J.L.; Lodge, C.J.; Lowe, A.J.; Campbell, B.E.; Matheson, M.C.; Hamilton, G.S.; Dharmage, S.C. Prevalence of obstructive sleep apnea in the general population: A systematic review. Sleep Med. Rev. 2017, 34, 70-81. [CrossRef]

8. Ong, J.C.; Crawford, M.; Dawson, S.C.; Fogg, L.F.; Turner, A.D.; Wyatt, J.K.; Crisostomo, M.I.; Chhangani, B.S.; Kushida, C.A.; Edinger, J.D.; et al. A randomized controlled trial of CBT-I and PAP for obstructive sleep apnea and comorbid insomnia: Main outcomes from the MATRICS study. Sleep 2020, 43, zsaa041. [CrossRef]

9. Sweetman, A.; Lack, L.; McEvoy, R.D.; Smith, S.; Eckert, D.J.; Osman, A.; Carberry, J.C.; Wallace, D.; Nguyen, P.D.; Catcheside, P. Bi-directional relationships between co-morbid insomnia and sleep apnea (COMISA). Sleep Med. Rev. 2021, 60, 101519. [CrossRef]

10. Zhang, Y.; Ren, R.; Lei, F.; Zhou, J.; Zhang, J.; Wing, Y.K.; Sanford, L.D.; Tang, X. Worldwide and regional prevalence rates of co-occurrence of insomnia and insomnia symptoms with obstructive sleep apnea: A systematic review and meta-analysis. Sleep Med. Rev. 2019, 45, 1-17. [CrossRef]

11. Sweetman, A.; Lack, L.; Bastien, C. Co-morbid insomnia and sleep apnea (COMISA): Prevalence, consequences, methodological considerations, and recent randomized controlled trials. Brain Sci. Sci. 2019, 9, 371. [CrossRef]

12. Lechat, B.; Appleton, S.; Melaku, A.Y.; Hansen, K.; McEvoy, D.R.; Adams, R.; Catcheside, P.; Lack, L.; Eckert, J.D.; Sweetman, A. Co-morbid insomnia and obstructive sleep apnoea is associated with all-cause mortality. Eur. Respir. J. 2021, 2101958. [CrossRef] [PubMed]

13. Mercer, D.J.; Bootzin, R.R.; Lack, L.C. Insomniacs' perception of wake instead of sleep. Sleep 2002, 25, 559-566. [CrossRef]

14. Crönlein, T.; Lehner, A.; Schüssler, P.; Geisler, P.; Rupprecht, R.; Wetter, T.C. Changes in subjective-objective sleep discrepancy following inpatient cognitive behavior therapy for insomnia. Behav. Ther. 2019, 50, 994-1001. [CrossRef]

15. Edinger, D.J.; Krystal, A.D. Subtyping primary insomnia: Is sleep state misperception a distinct clinical entity? Sleep Med. Rev. 2003, 7, 203-214. [CrossRef] [PubMed]

16. Lee, J.-W.; Choi, J.-H.; Kim, S.-G. Prevalence and Characteristics of Subjects with Obstructive Sleep Apnea among Adults with Insomnia Disorder. Sleep Med. Res. 2019, 10, 108-112. [CrossRef]

17. Tang, K.N.; Harvey, A.G. Correcting distorted perception of sleep in insomnia: A novel behavioural experiment? Behav. Res. Ther. 2004, 42, 27-39. [CrossRef]

18. Edinger, D.J.; Fins, A.I. The distribution and clinical significance of sleep time misperceptions among insomniacs. Sleep 1995, 18, 232-239. [CrossRef] 
19. Schutte-Rodin, S.; Broch, L.; Buysse, D.; Dorsey, C.; Sateia, M. Clinical guideline for the evaluation and management of chronic insomnia in adults. J. Clin. Sleep Med. 2008, 4, 487-504. [CrossRef]

20. Bootzin, R.R. Stimulus Control Treatment for Insomnia. Proc. Am. Psychol. Assoc. 1972, 7, 395-396.

21. Spielman, J.A.; Saskin, P.; Thorpy, M.J. Treatment of chronic insomnia by restriction of time in bed. Sleep 1987, 10, 45-56.

22. Harvey, A.G. A cognitive model of insomnia. Behav. Res. Ther. 2002, 40, 869-893. [CrossRef]

23. Lund, H.G.; Rybarczyk, B.D.; Perrin, P.B.; Leszczyszyn, D.; Stepanski, E. The discrepancy between subjective and objective measures of sleep in older adults receiving CBT for comorbid insomnia. J. Clin. Psychol. 2012, 69, 1108-1120. [CrossRef] [PubMed]

24. Mitchell, L.J.; Bisdounis, L.; Ballesio, A.; Omlin, X.; Kyle, S.D. The impact of cognitive behavioural therapy for insomnia on objective sleep parameters: A meta-analysis and systematic review. Sleep Med. Rev. 2019, 47, 90-102. [CrossRef] [PubMed]

25. Janků, K.; Šmotek, M.; Fárková, E.; Kopřivová, J. Subjective-objective sleep discrepancy in patients with insomnia during and after cognitive behavioural therapy: An actigraphy study. J. Sleep Res. 2020, 29, e13064. [CrossRef]

26. Sweetman, A.; Lack, L.; McEvoy, R.D.; Antic, N.A.; Smith, S.; Chai-Coetzer, C.L.; Douglas, J.; O'Grady, A.; Dunn, N.; Robinson, J.; et al. Cognitive behavioural therapy for insomnia reduces sleep apnoea severity: A randomised controlled trial. ERJ Open Res. 2020, 6, 00161-02020. [CrossRef] [PubMed]

27. Ratnavadivel, R.; Chau, N.; Stadler, D.; Yeo, A.; McEvoy, R.D.; Catcheside, P. Marked reduction in obstructive sleep apnea severity in slow wave sleep. J. Clin. Sleep Med. 2009, 5, 519-524. [CrossRef]

28. Bianchi, M.T.; Williams, K.L.; Mckinney, S.; Ellenbogen, J.M. The subjective-objective mismatch in sleep perception among those with insomnia and sleep apnea. J. Sleep Res. 2013, 22, 557-568. [CrossRef]

29. Choi, S.J.; Suh, S.; Ong, J.; Joo, E.Y. Sleep misperception in chronic insomnia patients with obstructive sleep apnea syndrome: Implications for clinical assessment. J. Clin. Sleep Med. 2016, 12, 1517-1525. [CrossRef]

30. Ma, Y.; Goldstein, M.R.; Davis, R.B.; Yeh, G.Y. Profile of subjective-objective sleep discrepancy in patients with insomnia and sleep apnea. J. Clin. Sleep Med. 2021, 17, 2155-2163. [CrossRef]

31. Sweetman, A.; Lack, L.; Catcheside, P.G.; Antic, A.N.; Smith, S.; Chai-Coetzer, C.L.; Douglas, J.; O'grady, A.; Dunn, N.; Robinson, J.; et al. Cognitive and behavioral therapy for insomnia increases the use of continuous positive airway pressure therapy in obstructive sleep apnea participants with comorbid insomnia: A randomized clinical trial. Sleep 2019, 42, 178. [CrossRef]

32. Iber, C. The AASM Manual for the Scoring of Sleep and Associated Events: Rules; Terminology and Technical Specification: Darien, IL, USA, 2007.

33. Ruehland, W.R.; Rochford, P.D.; O'donoghue, F.J.; Pierce, R.J.; Singh, P.; Thornton, A.T. The new AASM criteria for scoring hypopneas: Impact on the apnea hypopnea index. Sleep 2009, 32, 150-157. [CrossRef] [PubMed]

34. Liu, Y.; Tan, H.; Yu, Y.; Zeng, Y.; Xiao, L. Analysis of Clinical Characteristics and Polysomnography Indicators of Obstructive Sleep Apnea-Hypopnea Syndrome Patients Based on Sleep Perception Types. Front. Neurol. 2020, 11, 988. [CrossRef]

35. Bastien, C.H.; Vallières, A.; Morin, C.M. Validation of the Insomnia Severity Index as an outcome measure for insomnia research. Sleep Med. 2001, 2, 297-307. [CrossRef]

36. Gradisar, M.; Lack, L.; Harris, J.; Richards, H.; Gallasch, J.; Boundy, M.; Garrett, A. Psychometric properties of two new scales for measuring daytime functioning for insomnia. In Proceedings of the 20th Anniversary Meeting of the Associated Professional Sleep Societies, Salt Lake City, UT, USA, 17-22 June 2006.

37. Gradisar, M.; Lack, L.; Richards, H.; Harris, J.; Gallasch, J.; Boundy, M.; Johnston, A. The Flinders Fatigue Scale: Preliminary psychometric properties and clinical sensitivity of a new scale for measuring daytime fatigue associated with insomnia. J. Clin. Sleep Med. 2007, 3, 722-728. [CrossRef] [PubMed]

38. Cameron, K.; Williamson, P.; Short, M.A.; Gradisar, M. Validation of the Flinders Fatigue Scale as a measure of daytime fatigue. Sleep Med. 2017, 30, 105-112. [CrossRef]

39. Lovibond, H.S.; Lovibond, P.F. Manual for the Depression Anxiety Stress Scales; Psychology Foundation of Australia: Sydney, NSW, Australia, 1996.

40. Antony, M.M.; Antony, M.M.; Bieling, J.P.; Cox, J.B.; Enns, W.M.; Swinson, P.R. Psychometric properties of the 42-item and 21-item versions of the Depression Anxiety Stress Scales in clinical groups and a community sample. Psychol. Assess. 1998, 10, 176. [CrossRef]

41. Johns, M.W. A new method for measuring daytime sleepiness: The Epworth sleepiness scale. Sleep 1991, 14, 540-545. [CrossRef]

42. Johns, M.W. Reliability and factor analysis of the Epworth Sleepiness Scale. Sleep 1991, 14, 540-545. [CrossRef]

43. Morin, M.C.; Vallières, A.; Ivers, H. Dysfunctional beliefs and attitudes about sleep (DBAS): Validation of a brief version (DBAS-16). Sleep 2007, 30, 1547-1554. [CrossRef]

44. Manconi, M.; Ferri, R.; Sagrada, C.; Punjabi, N.M.; Tettamanzi, E.; Zucconi, M.; Oldani, A.; Castronovo, V.; Strambi, L.F. Measuring the error in sleep estimation in normal subjects and in patients with insomnia. J. Sleep Res. 2010, 19, 478-486. [CrossRef]

45. Hauri, J.P.; Olmstead, E.M. Reverse first night effect in insomnia. Sleep 1989, 12, 97-105. [CrossRef] [PubMed]

46. Kay, D.B.; Buysse, D.J.; Germain, A.; Hall, M.; Monk, T.H. Subjective-objective sleep discrepancy among older adults: Associations with insomnia diagnosis and insomnia treatment. J. Sleep Res. 2015, 24, 32-39. [CrossRef] [PubMed]

47. Dzierzewski, J.M.; Martin, J.L.; Fung, C.H.; Song, Y.; Fiorentino, L.; Jouldjian, S.; Rodriguez, J.C.; Mitchell, M.; Josephson, K.; Alessi, C.A. CBT for late-life insomnia and the accuracy of sleep and wake perceptions: Results from a randomized-controlled trial. J. Sleep Res. 2019, 28, e12809. [CrossRef] [PubMed] 
48. Nishikawa, K.; Kuriyama, K.; Yoshiike, T.; Yoshimura, A.; Okawa, M.; Kadotani, H.; Yamada, N. Effects of Cognitive Behavioral Therapy for Insomnia on Subjective-Objective Sleep Discrepancy in Patients with Primary Insomnia: A Small-Scale Cohort Pilot Study. Int. J. Behav. Med. 2021, 28, 1-12. [CrossRef] [PubMed]

49. Chan, W.S.; Dautovich, N.D.; McNamara, J.P.; Stripling, A.; Dzietrzewski, J.M.; McCoy, K.; McCrale, C.S. Sleep discrepancy in a randomized controlled trial of brief behavioral therapy for chronic insomnia in older adults. Behav. Sleep Med. 2021, 19, $221-231$. [CrossRef] 